Article

\title{
Polymerization Parameters Influencing the QCM Response Characteristics of BSA MIP
}

\author{
Nam V. H. Phan, Hermann F. Sussitz and Peter A. Lieberzeit* \\ Department of Analytical Chemistry, Faculty for Chemistry, University of Vienna, \\ Währinger Straße 38, A-1090 Vienna, Austria; E-Mails: honamd99@yahoo.com (N.V.H.P.); \\ hermann.franz.sussitz@univie.ac.at (H.F.S.)
}

* Author to whom correspondence should be addressed; E-Mail: peter.lieberzeit@univie.ac.at; Tel.: +43-1-4277-523-41.

Received: 8 April 2014; in revised form: 23 May 2014 / Accepted: 9 June 2014 /

Published: 16 June 2014

\begin{abstract}
Designing Molecularly Imprinted Polymers for sensing proteins is still a somewhat empirical process due to the inherent complexity of protein imprinting. Based on Bovine Serum Albumin as a model analyte, we explored the influence of a range of experimental parameters on the final sensor responses. The optimized polymer contains $70 \%$ cross linker. Lower amounts lead to higher sensitivity, but also sensor response times substantially increase (to up to $10 \mathrm{~h}$ ) at constant imprinting effect (signal ratio MIP/NIP on quartz crystal microbalance-QCM). However, by shifting the polymer properties to more hydrophilic by replacing methacrylic acid by acrylic acid, part of the decreased sensitivity can be recovered leading to appreciable sensor responses. Changing polymer morphology by bulk imprinting and nanoparticle approaches has much lower influence on sensitivity.
\end{abstract}

Keywords: molecular imprinted polymer; protein imprinting; polymer optimization; QCM

\section{Introduction}

Recent years have seen very strong increase in everyday application of biotechnology products as well as diagnostics shifting from centralized laboratories to distributed measurements. This also increases the need for quality control and suitable receptors for the respective sensor systems. Natural recognition systems fit perfectly in terms of sensitivity. However, in terms of technological application they are somewhat limited concerning stability and costs. Therefore, artificial recognition systems such 
as molecularly imprinted polymers (MIP) have attracted increasing attention. These materials aim to mimic natural recognition principles such as antibody-antigen interactions [1,2]. Imprinted polymers are typically cheap, straightforward to generate and effective in creating recognition systems for various types of analytes. Among others, MIP can be combined with mass-sensitive quartz micro balances (QCM) [3] to create powerful sensors for various types of analytes [4-6] that can also be applied in real life conditions [7]. Although several imprinting techniques for creating protein MIPs have been developed [8], there is not yet a systematic procedure for protein imprinting. This can be explained by several challenges one faces in protein imprinting [9]: Diffusion of larger bio species into the polymer is inhibited. Proteins can change their steric properties depending on $\mathrm{pH}$ and temperature. Due to this fact the steric recognition property of MIP might be lost. Additionally, proteins of course are large molecules containing many functional groups on their surfaces. Depending on the exact orientation during imprinting they can result in a large number of different binding sites on their respective surface area with broadly distributed affinity. Also protein imprinting is mainly limited to aqueous solution, where water competes for the corresponding binding sites with the analyte [10]. Considering this, rationally optimizing those polymers is the first important step on the way to protein MIP sensors. The aim of this paper is to systematically examine various polymer properties, such as cross linker ratio and functional monomer, and their influence on sensitivity and response time. For this purpose Bovine Serum Albumin (BSA) was chosen as model protein. BSA is a globular protein and extensively described in literature [11]. We hence prepared albumin-imprinted polymers for mass-sensitive sensing. All sensor responses of imprinted polymers are compared to a non-imprinted polymer (NIP) to compensate for any physical influence or non-specific adsorption on the surface. As MIP and NIP are chemically identical, any difference in their respective sensor signals only correlates to the imprinting effect. A scheme of such a QCM coated with MIP and NIP, respectively, is shown in Figure 1.

Figure 1. Scheme of a two-electrode quartz coated with two different polymers; molecularly imprinted polymer (MIP) and non-imprinted polymer (NIP).

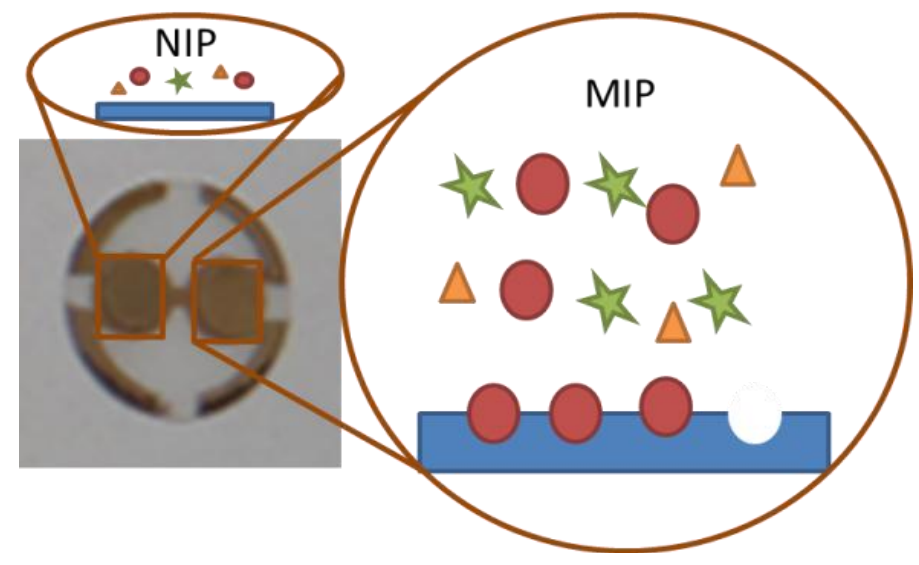

\section{Experimental Section}

\subsection{Materials and Devices}

Two-electrode $10 \mathrm{MHz}$ QCM were prepared via screen printing of brilliant gold paste (Hereus; $12 \%$ ) and removing organic residues at $400{ }^{\circ} \mathrm{C}$ for $4 \mathrm{~h}$. Afterwards, damping and resonance frequency 
were determined with an Agilent 8712ET network analyzer. The respective electrodes with a diameter of $5 \mathrm{~mm}$ were coated with MIP and NIP and the QCM was mounted into a measurement cell, which was connected to an oscillator circuit. We applied Agilent 53151A frequency counters do determine oscillator frequency as a function of time (the actual sensor signal) except during washing with $0.1 \mathrm{M}$ PBS, $0.1 \%$-SDS and water $10 \mathrm{~min}$ each, when read-out was paused. No negative influence of the washing step on the BSA rebinding properties of the sensors could be observed. The overall scheme of this setup is shown in Figure 2. Atomic force microscopy (AFM) measurements took place on a Bruker Instruments Nanoscope 8. Measurements were performed in tapping mode, a TESPA tip was used.

Figure 2. Measurement setup consisting of measuring cell, oscillator circuit and frequency counter. The thermostated solution was pumped through the measuring cell $(0.46 \mathrm{~mL} / \mathrm{min})$.

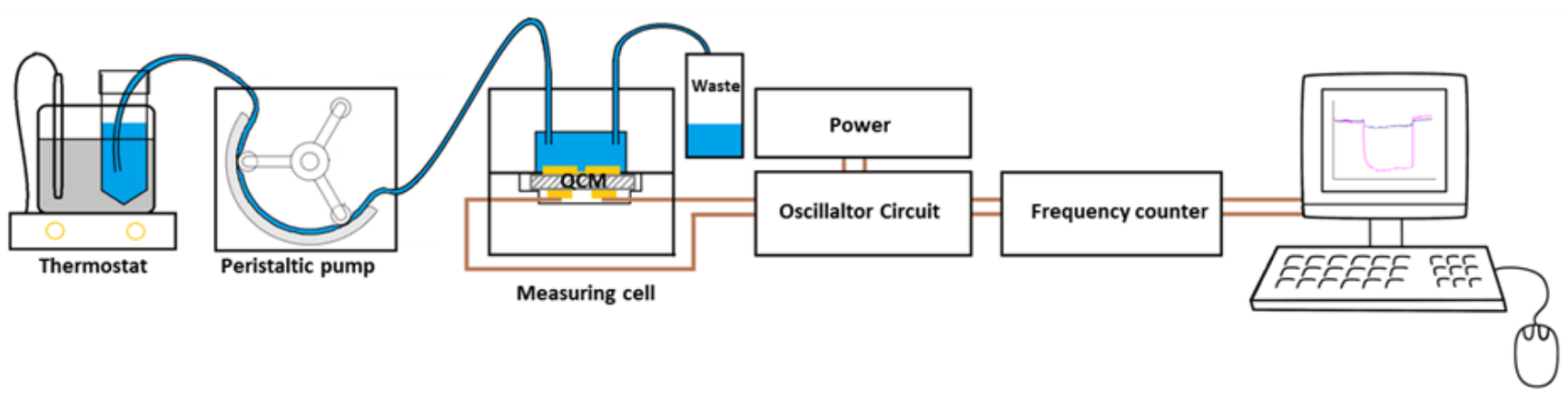

Flow rate required optimization: if chosen too high, noise increases, whereas low flow rate will favor sedimentation and thus lead to false positive signals due to mass change without binding to the selective layer. For these experiments $0.46 \mathrm{~mL} / \mathrm{min}$ turned out optimal. BSA was purchased at Sigma Aldrich, lyophilized powder $\geq 98 \%$. All other chemicals were purchased at VWR or Sigma Aldrich in highest available purity. $100 \mathrm{mM}$ PBS Buffer was prepared by diluting $80 \mathrm{~g} \mathrm{NaCl}, 2 \mathrm{~g} \mathrm{KCl}, 14.4 \mathrm{~g}$ $\mathrm{Na}_{2} \mathrm{HPO}_{4}$ and $2.4 \mathrm{~g} \mathrm{KH}_{2} \mathrm{PO}_{4}$ in $1 \mathrm{~L}$ water adjusted to $\mathrm{pH} 7.4$ with $\mathrm{HCl}$.

\subsection{Experimental}

The general synthetic strategy for obtaining MIP was as follows: First cross linker, functional monomer(s) and the radical starter (potassium peroxidisulfate; $1 \mathrm{mg}$ added per $\mathrm{mL}$ monomer solution) were dissolved in $1 \mathrm{~mL}$ Water and pre-polymerized under UV light. For the systematic studies of polymers N,N'-(1,2-dihydroxyethylene)bisacrylamide (DHEBA) was used as the cross linker, and methacrylic acid (MAA), acrylic acid (AA) and vinylpyrrolidone (VP) as functional monomers in different ratios. Details of the exact polymer compositions are summarized in Section 3. Depending on polymer composition the translucent solutions turned opaque after approximately 15-30 min indicating successful pre-polymerization. The pre-polymerized solution was spin-coated $5 \mathrm{~s}$ with $2000 \mathrm{rpm}$ onto the electrodes. For stamp imprinting we prepared albumin stamps on $5 \times 5 \mathrm{~mm}$ microscope slides. For that purpose we incubated slides in BSA solution for $1 \mathrm{~h}$ at $4{ }^{\circ} \mathrm{C}$. Following that, the BSA stamp was pressed into the oligomer deposited onto one of the QCM electrodes. After hardening for $48 \mathrm{~h}$ at room temperature, the stamp was removed by washing with water. In order to remove the template the polymer was washed with $100 \mathrm{mM}$ PBS-Buffer, 0.1\%-sodium dodecyl sulphate (SDS) and water $10 \mathrm{~min}$ each. In this way we obtained layers that were about $250 \mathrm{~nm}$ thick as determined by network analyzer and AFM. 
In addition to stamp imprinting we also developed a modified bulk imprinting process. This method has already been described for small molecules [12], however to the best of our knowledge it has not yet been tested on larger bio-species. Pre-polymerization took place as described before but for a minor change: the ingredients were dissolved in $950 \mu \mathrm{L}$ Water. The oligomer solution was then cooled to room temperature in a water bath, afterwards $50 \mu \mathrm{L} 40 \mathrm{mg} / \mathrm{mL}$ Albumin solution was added for the MIP and $50 \mu \mathrm{L}$ water for the NIP. After 15 min self-organization, oligomer solutions were spin-coated onto the two corresponding electrodes at $2000 \mathrm{rpm}$ for $30 \mathrm{~s}$ followed by removing the template from the polymer as described before.

Furthermore, we also prepared MIP nanoparticles. For that purpose, the above mentioned oligomer solutions served to synthesize polymer nanoparticles via precipitation [13]: the solution was added to $15 \mathrm{~mL}$ acetonitrile and stirred vigorously for $48 \mathrm{~h}$. The resulting particles were centrifuged, and the supernatant discarded. Finally, the nanoparticles were re-suspended in $1 \mathrm{~mL}$ acetonitrile. The concentrated solution was spin-coated onto the gold electrodes at $2000 \mathrm{rpm}$ for $30 \mathrm{~s}$, after $48 \mathrm{~h}$ the surface was washed as described before. To determine nanoparticle diameters the solution was diluted 1:10, spin coated onto a glass slide, dried for $24 \mathrm{~h}$ at ambient conditions and measured via atomic force microscopy.

\section{Results and Discussion}

\subsection{Polymer Optimization: Cross Linker}

In a first step we assessed the influence of the cross linker ratio on sensor responses. As a starting point for optimization, we prepared a polymer with a cross linker ratio of $30 \%$ (w/w) by mixing $15 \mathrm{mg}$ DHEBA, $25 \mathrm{mg}$ MAA and $10 \mathrm{mg}$ vinylpyrrolidone. One corresponding QCM signal is shown in Figure 3. After equilibration to achieve constant frequency with a noise level around 5-7 Hz, we added a solution of $100 \mathrm{mg} / \mathrm{mL}$ albumin in PBS buffer, which leads to decreasing frequency. However, it took $10 \mathrm{~h}$ for the signal to reach equilibrium. The frequency difference between $900 \mathrm{~Hz}$ for MIP and $500 \mathrm{~Hz}$ for NIP corresponds to $400 \mathrm{~Hz}$ sensor signal for $100 \mathrm{mg} / \mathrm{L}$ Albumin as a consequence of the imprinting process. After washing with water, the measurement was resumed until the signal reached baseline again. Such full reversibility proves both successful washing and full removal of the analyte. In the case of $30 \%$ cross linker swelling of the polymer is probable, which usually leads to signal drift, when the signals for both channels change continuously. As reaching stable frequencies could still have lasted for hours without providing further insights, the experiment was stopped. Also at $30 \%$ cross linker the mobility of the polymer chain is the highest; therefore some polymer or oligomer can be removed during washing, which explains the slight baseline overshoot in Figure 3.

Repeated measurement with the same quartz revealed that the sensor signal remains basically constant, namely $470 \mathrm{~Hz}$. However the response time increased to $16 \mathrm{~h}$. This effect indicates less pronounced interaction sites, which can be explained by cross-linker cleavage by slightly alkaline washing solutions. During further experiments the monomer ratio (MAA:VP) was kept constant at 5:2 (w/w), while the cross-linker ratio was increased up to $70 \%(\mathrm{w} / \mathrm{w})$. The corresponding polymer compositions are summarized in Table 1 below. Polymers with cross-linker ratios of 40, 50, 60 and $70 \%(\mathrm{w} / \mathrm{w})$ were prepared and measured as described before. The respective results are shown in Figure 4. The sensors were characterized in terms of sensor signal and response times. 
Figure 3. Quartz micro balance (QCM) sensor responses of $100 \mathrm{mg} / \mathrm{L}$ albumin.

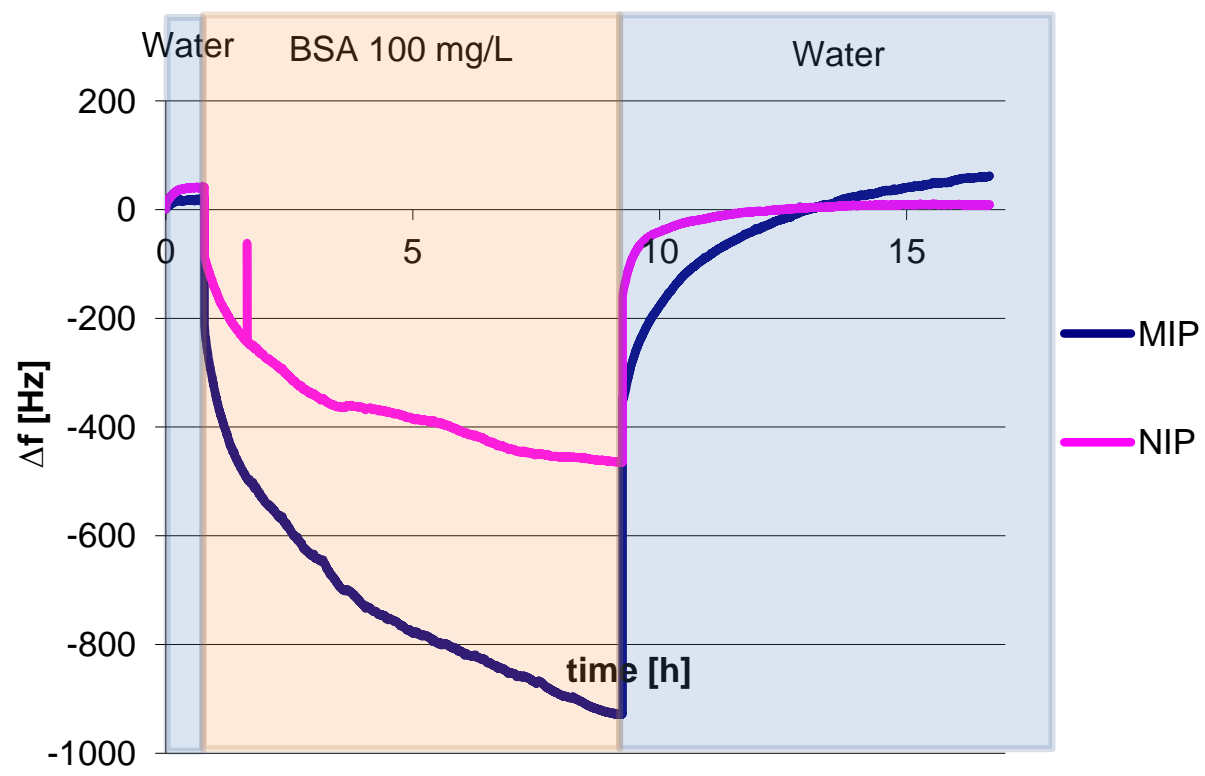

Table 1. Polymer compositions with different amount of cross linker.

\begin{tabular}{cccccc}
\hline Cross-linker (\%) & $\mathbf{3 0 \%}$ & $\mathbf{4 0 \%}$ & $\mathbf{5 0 \%}$ & $\mathbf{6 0 \%}$ & $\mathbf{7 0 \%}$ \\
\hline mg & 15 & 15 & 25 & 30 & 35 \\
Monomers (mg) & 35 & 17.5 & 25 & 20 & 15 \\
\hline
\end{tabular}

Figure 4. Sensor signals for MIP containing different amounts of cross linker (w/w); functional monomers (MAA:VP; 5:2 w/w).

Influence of cross linker ratio to BSA sensor signal $(100 \mathrm{mg} / \mathrm{L})$

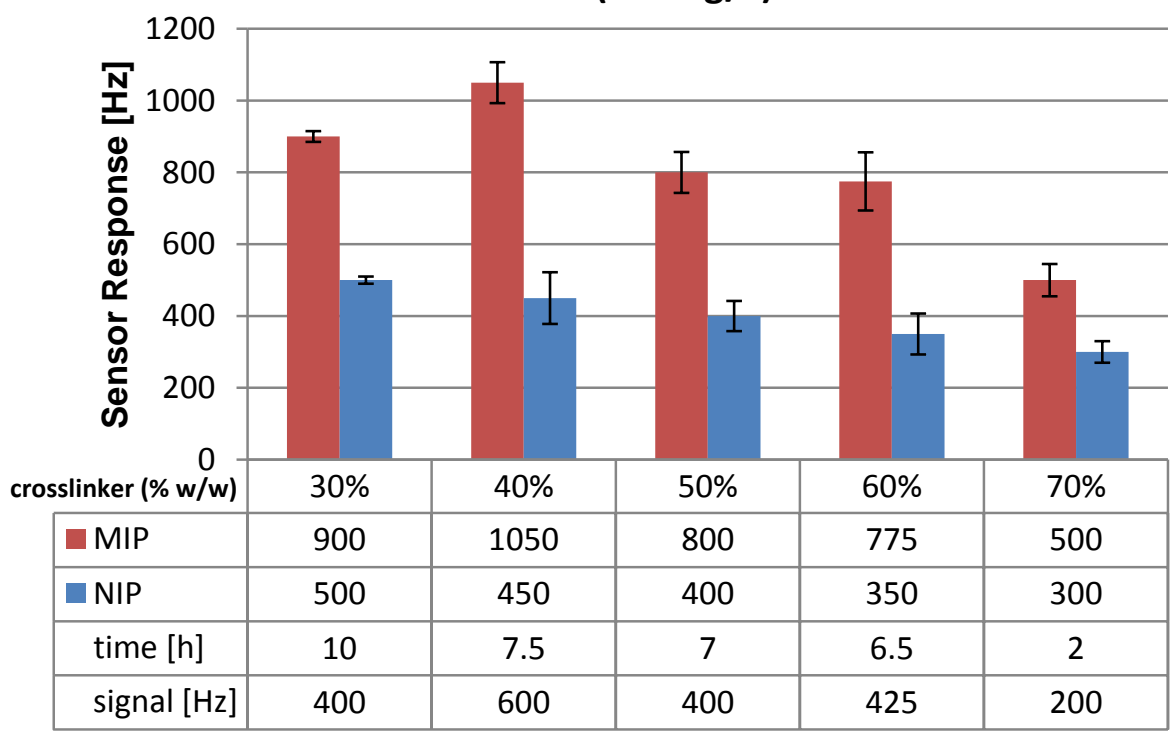

The highest sensor signal of $600 \mathrm{~Hz}$ was obtained with $40 \%(\mathrm{w} / \mathrm{w})$ cross-linker, while $50 \%$ and $60 \%$ cross-linker $(\mathrm{w} / \mathrm{w})$ showed similar results to $30 \%$. When the cross-linker ratio is increased from 60 to $70 \%$ the sensor signal was reduced by a factor of 2 from 425 to $200 \mathrm{~Hz}$. This seems to contradict 
results obtained with small molecules, where increase of cross linker leads to an increase in sensitivity [14]. However, the main difference between the two systems is that BSA imprints are only present on the surface of the sensor layer and not the full bulk of the film. Polymers with lower cross linker ratio are expected to swell because of the higher mobility of polymer chains. This leads to gel-like structures for which the Sauerbery equation is no longer fully valid. The slow sensor responses in combination with high sensitivity on both polymers (MIP and NIP) indicate that this may lead to adaptive processes between the film and the BSA solution.

Furthermore response time strongly depends on the amount of cross-linker: The higher the amount of cross linker, the lower response times become. For instance, when going from 30\% to $70 \%$ cross-linker, the time for the sensors to reach equilibrium is reduced by a factor of five from 10 to $2 \mathrm{~h}$. This can also be explained by a more rigid polymer where the surface is more clearly defined compared to a soft polymer. Due to increased cross linker ratio, fewer functional monomer binding sites are present on the surface.

\subsection{Polymer Optimization: Functional Monomer}

A cross-linker ratio of $70 \%$ was chosen for further systematic studies of functional monomers to ensure appreciably rapid sensing events. The different polymer compositions for assessing the role of functional monomers are described in detail in Table 2.

Table 2. Polymer compositions with a constant ratio of cross linker $(70 \% \mathrm{w} / \mathrm{w})$.

\begin{tabular}{lccccc}
\hline & Name & cross linker & $\begin{array}{c}\text { functional } \\
\text { monomer 1 }\end{array}$ & $\begin{array}{c}\text { functional } \\
\text { monomer 2 }\end{array}$ & $\begin{array}{c}\text { functional } \\
\text { monomer ratio w/w }\end{array}$ \\
\hline $\begin{array}{l}\text { ratio } \\
(\mathbf{w} / \mathbf{w})\end{array}$ & - & \multicolumn{3}{c}{$30 \%$} & - \\
& MA 1 & DHEBA & methacrylic acid & vinylpyrrolidone & $5: 2$ \\
& AA 1 & DHEBA & acrylic acid & vinylpyrrolidone & $5: 2$ \\
& AA 2 & DHEBA & acrylic acid & vinylpyrrolidone & $1: 1$ \\
& AA 3 & DHEBA & acrylic acid & vinylpyrrolidone & $2: 3$ \\
\hline
\end{tabular}

The outcome for all these materials is summarized in Figure 5. Sensitivity could be increased significantly by using acrylic acid as a monomer rather than methacrylic acid: sensor responses went from $200 \mathrm{~Hz}$ to $900 \mathrm{~Hz}$ and thus increased by a factor of 4.5. However the response times increased by roughly the same factor. Varying the ratio of the two functional monomers (acrylic acid and vinyl pyrrolidone) has small, but highly significant effects: Functional monomer ratio of 1:1 (w/w) AA:VP leads to a sensor signal of $550 \mathrm{~Hz}$ while a ratio $2: 3$ (w/w) led to $355 \mathrm{~Hz}$. Increasing the amount of vinylpyrrolidone thus reduces sensitivity. However, response times also reached the initial value.

Compared to the first composition containing 30\% cross-linker and 5:2 MAA:VP functional monomer ratio, the sensor signal of $70 \%$ cross-linker with 2:3 AA:VP functional monomer is only 1.2 times lower, but the sensor response time decreased by a factor of 2.5. Therefore, the functional monomer ratio of 2:3 AA:VP with $70 \%$ cross-linker ratio fits perfectly in terms of sensor response and sensitivity. The selection of imprinting protocols has always relied on balancing different molecular properties such as combination of strong/dissociable (e.g., methacrylic acid) or others on weak/neutral binding monomers (e.g., acrylamide) for template recognition [15]. 
Figure 5. QCM sensor signals obtained for different ratios of functional monomers; cross linker $70 \%$. The sensor signal shown is the difference in response between MIP and NIP.

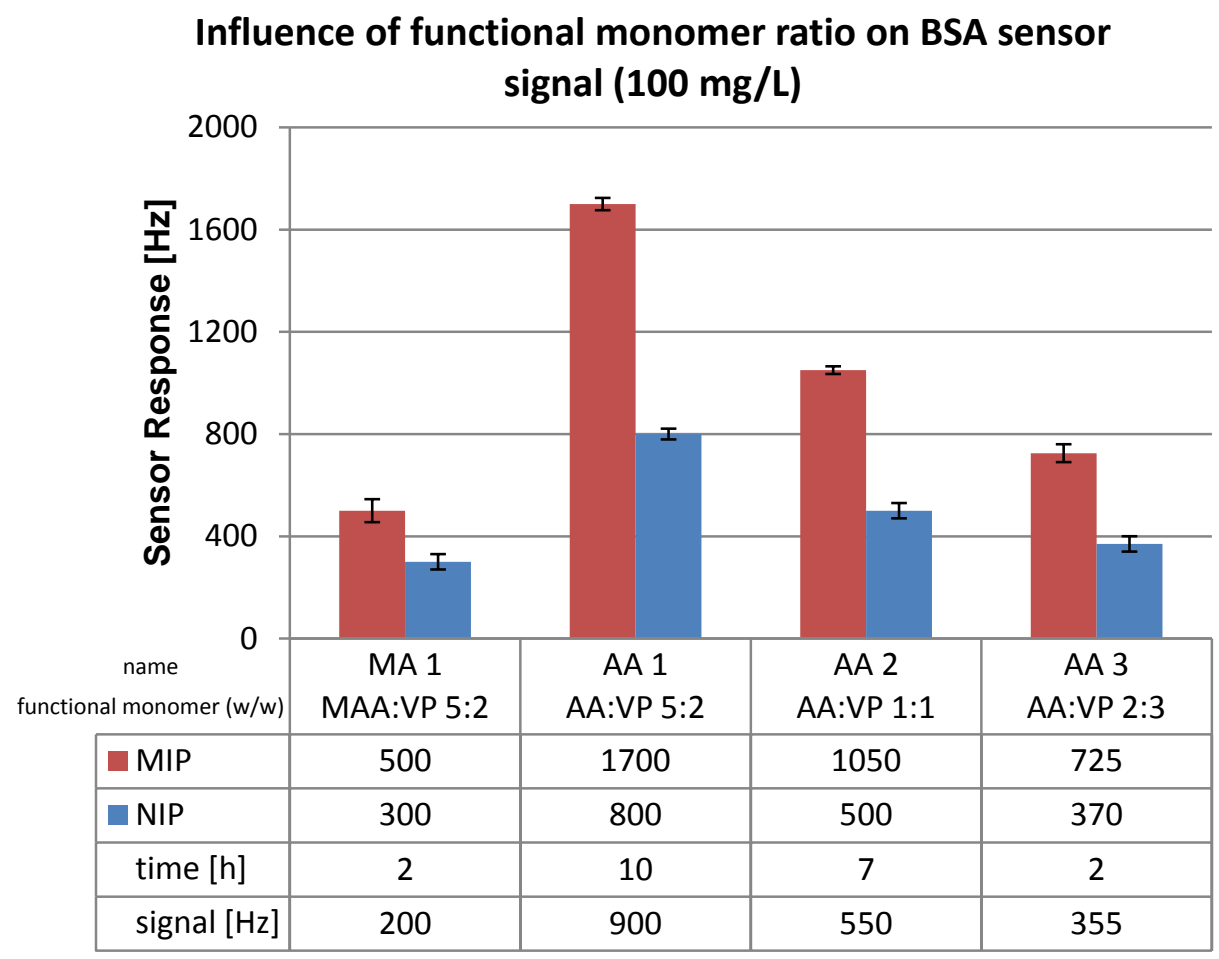

To obtain a sensor characteristic of an Albumin sensor, polymer AA3 with a cross linker ratio of $70 \%(\mathrm{w} / \mathrm{w})$ and a functional monomer ratio of 2:3 AA:VP was chosen. First measurements indicate that the acrylic acid is the driving force for the interaction between the protein and the polymer. When decreasing the functional monomer ratio of acrylic acid the interaction is reduced, therefore the sensor response signal is decreased. On the other hand the diffusion into the polymer seems to be enhanced by a higher amount of VP. It was possible to calibrate the sensor in a dynamic range ranging from 10 to $100 \mathrm{mg} / \mathrm{L}(0.15-1.5 \mu \mathrm{M} / \mathrm{L})$ as shown in Figure 6.

Figure 6. Sensor characteristic of the optimized polymer towards Albumin.

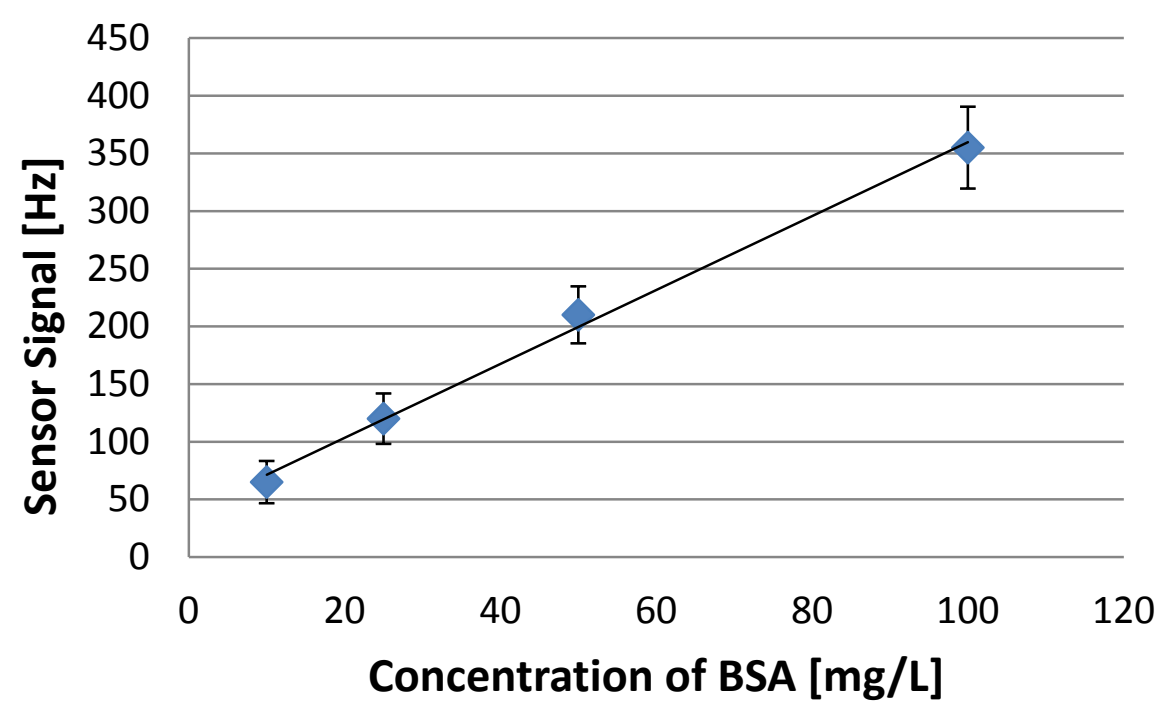


Sensor signals of $65 \mathrm{~Hz}$ for $10 \mathrm{mg} / \mathrm{L}, 120 \mathrm{~Hz}$ for $25 \mathrm{mg} / \mathrm{L}, 210 \mathrm{~Hz}$ for $50 \mathrm{mg} / \mathrm{L}$ and $355 \mathrm{~Hz}$ for $100 \mathrm{mg} / \mathrm{L}$ were obtained. The sensor shows a linear behavior. Generally noise is around 5-7 Hz so in theory a signal of $15 \mathrm{~Hz}$ could be determined which would mean a (theoretical) limit of detection of $5 \mathrm{mg} / \mathrm{L}$. In Literature LoD of $0.1 \mathrm{mg} / \mathrm{L}$ has been reported for Human Serum Albumin [16]. This detection limit is lower than the one for BSA reported here; however, it was obtained by antibody-antigen interaction. In general detection systems derived by nature are often more sensitive than their artificial counterparts, but they cannot be used under nonphysiological conditions and are far more expensive. Artificial recognition systems can hence be a cost-effective alternative in technological applications after optimization.

\subsection{Polymerization Solvent}

It is expected that polar aprotic media would be better for the polymerization process because water competes with the corresponding protein. To investigate the effect the same polymer was prepared in dimethylsulfoxide (DMSO). The sensor signal is reduced by a factor of 1.75 to $200 \mathrm{~Hz}$ compared to the same polymer composition prepared in water. Poor solubility of albumin in DMSO hence counteracts the signal increase achieved from optimization.

\subsection{Polymer Morphology}

Molecularly imprinted polymers can be achieved by various strategies, for instance stamp imprinting was chosen for BSA. However this approach does not necessarily ensure optimal self-organization of the analyte and the functional monomers. Bulk imprinting could be used as an alternative. However, proteins are not necessarily suitable for the harsh polymerization procedure at higher temperature or UV-light: Albumin denatures at $60{ }^{\circ} \mathrm{C}$, which is the temperature it would have to withstand during pre-polymerization for one hour. To solve this problem we modified bulk imprinting. This overcomes some disadvantages of stamp imprinting, such as damaging the surface during stamp removal. For the modified bulk imprinting approach we prepared the optimized polymer from $35 \mathrm{mg}$ DHEBA, $8 \mathrm{mg} \mathrm{VP}$ and $6 \mathrm{mg} \mathrm{AA}$ and polymerized in water following the procedures described in Section 2.1. Both methods, stamp MIP and modified bulk MIP, lead to similar response times - namely $2 \mathrm{~h}$ - and sensor signals (at $100 \mathrm{mg} / \mathrm{L}$ Albumin, $355 \mathrm{~Hz}$ for the stamp and $320 \mathrm{~Hz}$ for the modified bulk approach). Since signals (intensity, response time) are not influenced by the chosen polymerization strategy, systematically optimizing polymer composition evidently is the most important step during recognition layer design for QCM sensors.

Polymer morphology also can have some influence on sensor signals, because the number of available binding sites plays an important role for molecular recognition. For instance MIP nanoparticles lead to higher sensitivity in the case of small molecules as analytes due to high surface area and therefore increased amount of accessible binding sites. Nanoparticles are mainly prepared by micro emulsion polymerization [17]. This technique delivers well-defined particles in terms of size and shape. However, the modified bulk imprint approach described earlier makes it possible to create nanoparticles in water by precipitation methods thus substantially reducing the complexity of the polymerization system. Figure 7 shows an AFM image of BSA MIP nanoparticles obtained by such precipitation. Evidently, particles are roughly $350 \mathrm{~nm}$ in diameter and seem to be $40 \mathrm{~nm}$ thick. The 
reason for this observation, however, most probably is the fact that soft particles deposited on a surface behave in gel-like manner to form disk-shaped deposits.

Albumin nanoparticles on QCM revealed a 0.75 times lower signal, namely $277 \mathrm{~Hz}$, compared to MIP thin films resulting from the stamping approach. This can be explained by the large size distribution of the nanoparticles and the geometrical features: As mentioned, NP deposited on the surface are only 30 to $40 \mathrm{~nm}$ high and form spots that are 200 to $300 \mathrm{~nm}$ in diameter. This indicates that the nanoparticles are flattened during spin-coating. Generally clusters of nanoparticles or flattened particles will exhibit lower surface area than dispersed nanoparticles. Furthermore, the nanoparticles generated in this first attempt could easily be removed from the surface. Hence substantial further effort is necessary to optimize those structures to actually achieve the desired sensor properties.

Figure 7. Atomic force microscopy image of BSA MIP nanoparticles.
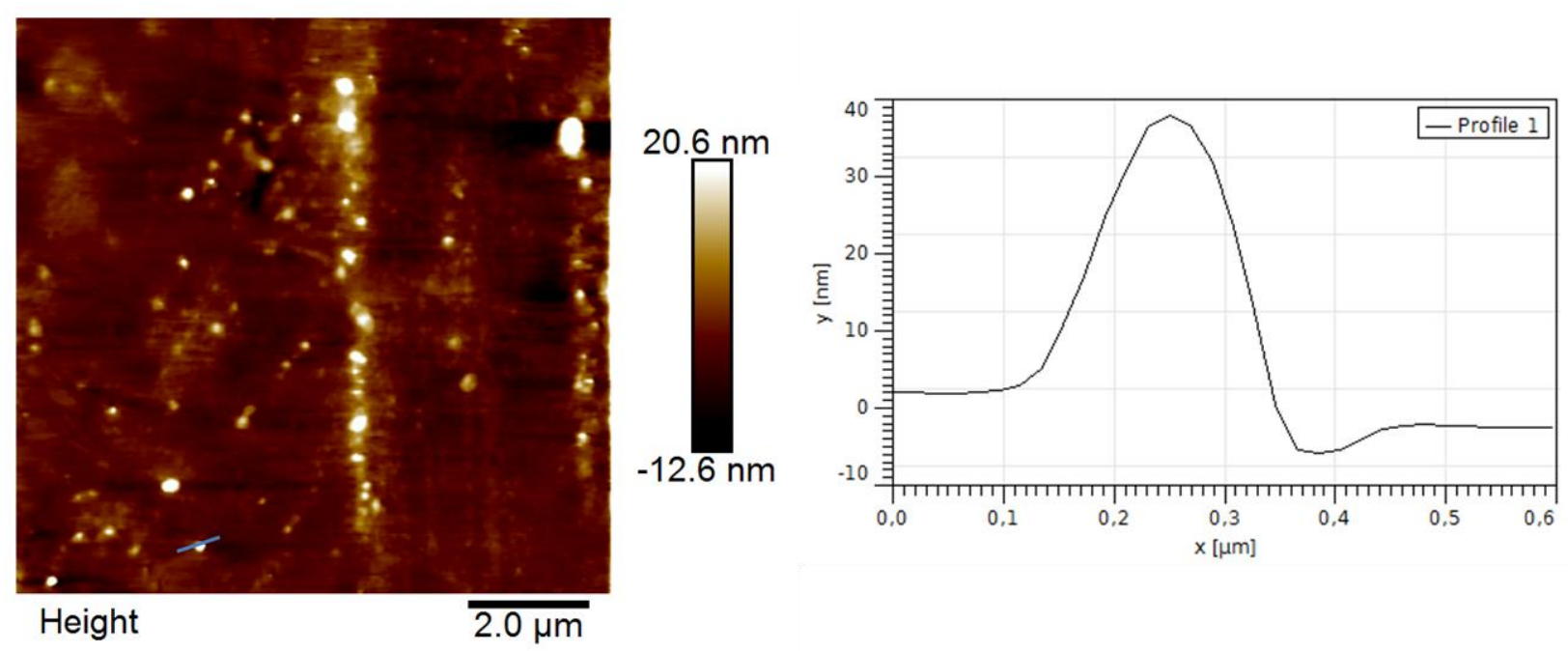

\section{Conclusions}

These optimization experiments on the way to a standardized stamping procedure for protein MIP revealed substantial influence of the ratio between cross linker and functional monomers: Sensor response time can be decreased by increasing the amount of cross-linker from $30 \%$ to $70 \%$. At the same time, also sensitivity decreases, which can be overcome by increasing the polarity of the sensor layer. Replacing methacrylic acid by acrylic acid leads to a higher sensor signal. This clearly shows the importance of optimizing polymer composition. Other parameters play a much smaller role in BSA MIP: replacing water as the polymerization solvent by DMSO, i.e., changing from polar protic to polar aprotic environment, decreases sensitivity despite the fact that this approach should support formation of hydrogen bonds between polymer and template. Polymer morphology also had much smaller influence on the sensor properties than polymer optimization: BSA MIP nanoparticle layers, for instance, lead to slightly lower sensor responses than the corresponding thin films (0.75 times).

\section{Acknowledgments}

NPVH gratefully acknowledges partial funding of his PhD studies by a TRIG project of the University of Medicine and Pharmacy, Ho Chi Minh City, Vietnam. 


\section{Author Contributions}

NPVH carried out experiments for BSA MIP optimization. HS developed the bulk imprinting technique and the MIP nanoparticles. PL supervised all work, gave scientific input and finalized the manuscript.

\section{Conflicts of Interest}

The authors declare no conflict of interest.

\section{References}

1. Kotova, K.; Hussain, M.; Mustafa, G.; Lieberzeit, P.A. MIP sensors on the way to biotech applications: Targeting selectivity. Sens. Actuator. B Chem. 2013, 189, 199-202.

2. Cakir, P.; Cutivet, A.; Resmini, M.; Bui, B.T.S.; Haupt, K. Protein-size molecularly imprinted polymer nanogels as synthetic antibodies, by localized polymerization with multi-initiators. Adv. Mater. 2013, 25, 1048-1051.

3. Sauerbrey, G. Verwendung Von Schwingquarzen zur wägung dünner Schichten und zur mikrowägung. Z Phys. 1959, 155, 206-222.

4. Nopper, D.; Lammershop, O.; Wulff, G.; Gauglitz, G. Amidine-based molecularly imprinted polymers-New sensitive elements for chiral chemosensors. Anal. Bioanal. Chem. 2003, 377, 608-613.

5. Alenus, J.; Ethirajan, A.; Horemans, F.; Weustenraed, A.; Csipai, P.; Gruber, J.; Peeters, M.; Cleij, T.J.; Wagner, P. Molecularly imprinted polymers as synthetic receptors for the QCM-D-based detection of L-nicotine in diluted saliva and urine samples. Anal. Bioanal. Chem. 2013, 405, 6479-6487.

6. Seidler, K.; Lieberzeit, P.A.; Dickert, F.L. Application of yeast imprinting in biotechnology and process control. Analyst 2009, 134, 361-366.

7. Lieberzeit, P.A.; Rehman, A.; Najafi, B.; Dickert, F.L. Real-life application of a QCM-based E-nose: Quantitative characterization different plant-degradation processes. Anal. Bioanal. Chem. 2008, 391, 2897-2903.

8. Turner, N.W.; Jeans, C.W.; Brain, K.R.; Allender, C.J.; Hlady, V.; Britt, D.W. From 3D to 2D: A review of the molecular imprinting of proteins. Biotechnol. Progr. 2006, 22, 1474-1489.

9. Mallik, S.; Plunkett, S.D.; Dhal, P.K.; Johnson, R.D.; Pack, D.; Shnek, D.; Arnold, F.H. Towards materials for the specific recognition and separation of proteins. New J. Chem. 1994, 18, 299-304.

10. Ramstrom, O; Ansell, R.J. Molecular imprinting technology: Challenges and prospects for the future. Chirality 1998, 10, 195-209.

11. Rothschild, M.A.; Oratz, M.; Schreiber, S.S. Serum-Albumin. Hepatology 1988, 8, 385-401.

12. Lieberzeit, P.A.; Rehman, A.; Yaqub, S.; Dickert, F.L. Nanostructured particles and layers for sensing contaminants in air and water. Nano 2008, 3, 205-208.

13. Yoshimatsu, K.; Reimhult, K.; Krozer, A.; Mosbach, K.; Sode, K.; Ye, L. Uniform molecularly imprinted microspheres and nanoparticles prepared by precipitation polymerization: The control of particle size suitable for different analytical applications. Anal. Chim. Acta 2007, 584, $112-121$. 
14. Vaidya, A.A.; Lele, B.S.; Kulkarni, M.G.; Mashelkar, R.A. Creating a macromolecular receptor by affinity imprinting. J. Appl. Polym. Sci. 2001, 81, 1075-1083.

15. Bossi, A.; Bonini, F.; Turner, A.P.F.; Piletsky, S.A. Molecularly imprinted polymers for the recognition of proteins: The state of the art. Biosens. Bioelectron. 2007, 22, 1131-1137.

16. Muratsugu, M.; Ohta, F.; Miya, Y.; Hosokawa, T.; Kurosawa, S.; Kamo, N.; Ikeda, H. Quartz-crystal microbalance for the detection of microgram quantities of human serum-albuminRelationship between the frequency change and the mass of protein adsorbed. Anal Chem 1993, 65, 2933-2937.

17. Poma, A.; Turner, A.P.F.; Piletsky, S.A. Advances in the manufacture of MIP nanoparticles. Trends Biotechnol. 2010, 28, 629-637.

(C) 2014 by the authors; licensee MDPI, Basel, Switzerland. This article is an open access article distributed under the terms and conditions of the Creative Commons Attribution license (http://creativecommons.org/licenses/by/3.0/). 